\title{
Mechanisms of Virtual Reality Exposure Therapy: The Role of the Behavioral Activation and Behavioral Inhibition Systems
}

\author{
Frank H. Wilhelm, ${ }^{1,5}$ Monique C. Pfaltz, ${ }^{1}$ James J. Gross, ${ }^{2}$ Iris B. Mauss, ${ }^{2}$ \\ Sun I. Kim, ${ }^{3}$ and Brenda K. Wiederhold ${ }^{4}$
}

\begin{abstract}
J. A. Gray's (1975) theory distinguishes between two motivational systems, which he refers to as the behavioral activation system (BAS) and the behavioral inhibition system (BIS). $D$. C. Fowles (1980) has shown that heart rate responses reflect activity of the BAS, and electrodermal responses reflect activity of the BIS. Both BAS and BIS are reliably activated during in-vivo exposure to fearful situations (F. H. Wilhelm \& W. T. Roth, 1998). However, due to the constraints imposed by virtual reality (VR), we hypothesized that VR exposure to fearful situations would activate the BIS alone. To test this hypothesis, a VR free-standing elevator simulation was presented to participants selected for high and low fear of heights. As predicted, the high-anxious group strongly responded electrodermally (effect size $d=1.53)$, but showed only minimal HR elevations during exposure $(d=0.12)$, and little other cardiovascular or respiratory changes. The low-anxious control group showed little electrodermal and HR reactivity $(d=0.28$ and 0.12$)$. A comparison with data from a previous study demonstrated that this pattern was in stark contrast to the large electrodermal and cardiovascular response observed during situational in-vivo exposure outside the laboratory. We conclude that the BIS, but not BAS, is selectively activated during $V R$ exposure, causing discordance between self-report and commonly used physiological measures of anxiety. Results are discussed within the framework of E. B. Foa \& M.J. Kozak's (1986) emotional processing theory of fear modification, suggesting different mechanisms underlying VR and in-vivo exposure treatments.
\end{abstract}

KEY WORDS: virtual reality; exposure; emotion; anxiety; phobia; cognitive-behavior therapy; heart rate; skin conductance; respiration.

Over the past several decades, in-vivo exposure therapy (IVET) has been shown to be an effective treatment for anxiety disorders (Barlow, 2002). With the emergence of virtual reality (VR) technology, clinicians have begun to examine the clinical efficacy of

\footnotetext{
${ }^{1}$ Institute for Psychology, University of Basel, Basel, Switzerland.

${ }^{2}$ Department of Psychology, Stanford University, Stanford, California.

${ }^{3}$ Department of Biomedical Engineering, College of Medicine, Hanyang University, Seoul, Korea.

${ }^{4}$ Virtual Reality Medical Center, Interactive Media Institute, San Diego, California.

${ }^{5}$ Address all correspondence to Frank H. Wilhelm, Health Psychophysiology Laboratory, Institute for Psychology, University of Basel, CH-4055 Basel, Switzerland; e-mail: frank.wilhelm@unibas.ch.
} 
VR exposure therapy (VRET). Findings of randomized clinical trials in which VRET is compared with standard exposure to date suggest that VRET may be used to successfully treat specific phobias of the situational type, such as acrophobia or fear of flying (Krijn, Emmelkamp, Olafsson, \& Biemond, 2004; Rothbaum, Hodges, Smith, Lee, \& Price, 2000; Rothbaum et al., 1995). It is not clear, however, whether the mechanisms underlying VRET are the same as those underlying IVET.

According to Foa and Kozak's (1986) emotional processing theory, exposure therapy ultimately establishes new memory structures that replace old, anxiety-provoking ones. Within this framework, physiological activation is seen as a byproduct of the activation of relevant fear structures during exposure therapy. This activation is believed to be advantageous for restructuring to occur. This suggests a crucial question: Do comparable physiological changes occur during in-vivo and VR exposure therapies?

Almost all IVET studies that measured physiological changes have examined heart rate (HR) as the sole index of physiological activation. Several of these studies have demonstrated that initial HR increase during in-vivo exposure predicts treatment success (Foa \& McNally, 1996). There have also been a few studies that have examined activation across a broader range of physiological systems during in-vivo exposure, including studies of fear of flying (Wilhelm \& Roth, 1998) and fear of driving (Alpers, Wilhelm, \& Roth, 2005). In these metric comparison studies, HR has been identified as a particularly strong measure of anxiety. It increased on average about 20-25 bpm in phobic individuals during exposure treatment. An index of clinical utility of an anxiety measure is its effect size for the strength of diagnostic discrimination, comparing phobic with control groups' reactivity during exposure. For HR, effect sizes ranged in the order of 1.4, which indicates potency even for individual diagnostic categorization. An electrodermal measure, skin conductance level (SCL), had effect sizes in the order of 0.8 , which makes it a less powerful diagnostic discriminator. In the flight phobia study, about $80 \%$ of phobics were correctly identified solely on the basis of their HR response, whereas only $65 \%$ were identified from their SCL response. Almost all phobic individuals (97\%) were correctly identified when a combination of five physiological measures was used.

With regard to VRET, such objective physiological measures of anxiety have not been established well. Most of the studies conducted to date rely on individual's subjective selfreports to assess levels of anxiety experienced during VRET (Krijn et al., 2004). Given the new therapeutic opportunities offered by this technology, physiological monitoring would seem to hold out clear promise to help objectively evaluate VRET outcomes and to better understand the underlying mechanisms (Maheu, Pulier, Wilhelm, McMenamin, \& Brown-Connolly, 2005). However, initial findings have been puzzling, indicating that HR responses during VRET are minimal or nonexistent, even when patients report high levels of fear (Wiederhold, Jang, Kim, \& Wiederhold, 2002). Electrodermal activity, on the other hand, seems to clearly distinguish phobic from non-phobic individuals. Why might this be?

One potential explanation is provided by Fowles' motivational theory (Fowles, 1980, 1988). According to this theory, situations that do not allow for active avoidance responses to fear-evoking stimuli selectively activate the electrodermal but not cardiovascular system. Fowles' approach is based on work by Gray, who, referring to the literature on animal learning and motivation, distinguished between an appetitive and an aversive motivational system (Gray, 1975). The appetitive motivational system, also referred to as the behavioral activation system (BAS), is involved in simple reward-approach situations, as well as in the 
initiation of behavior in active avoidance situations, in which the organism must respond to avoid punishment. The aversive motivational system, or behavioral inhibition system (BIS), is viewed as an anxiety system and inhibits appetitively motivated behavior in the presence of cues signaling that frustrative or anxiety evoking aversive consequences will occur in case the appetitively motivated behavior is shown. Passive avoidance is one situation that activates the BIS. In a series of studies, Fowles and colleagues demonstrated that HR is primarily influenced by the BAS, whereas SCL is primarily influenced by the BIS (Fowles, 1988).

In the present study, we examined the relative contributions of the motivational systems represented by the BAS and BIS during the course of a standardized VR height exposure session in individuals afraid of heights. We hypothesized that due to the constraining nature of VR technology in which no substantial motorically engaging active avoidance responses may be made, the BAS system would play a minor role. On the other hand, because the VR situation is characterized by physical passivity in the face of an anxiety-provoking environment, we hypothesized that the BIS system would be engaged. These hypotheses led us to predict that the electrodermal system should be the physiological system that is most responsive to VR challenges, while cardiovascular responses, including HR, will be activated only to a small degree. To better delineate nonspecific effects not related to anxiety, we contrasted responses of anxious individuals with those of non-anxious controls. A secondary aim of this research was to examine the relative clinical utility of a variety of cardiovascular and respiratory measures for discriminating between anxious and nonanxious individuals during VR exposure, quantified by their effect sizes. Finally, we were curious in how the pattern of physiological responses of the current study, especially pertaining to the BIS and BAS, compared to our previous study that used in-vivo exposure for participants with fear of flying (Wilhelm \& Roth, 1997, 1998).

\section{METHODS}

\section{Participants}

Participants were selected from a pool of 86 undergraduates using the Acrophobia Questionnaire (Cohen, 1977). This questionnaire describes 20 situations that can cause fear of heights (e.g., driving over a bridge, walking over a sidewalk grating, or sitting in an airplane) and asks for anxiety ratings on 7-point (0-6) Likert-type scales. Based on their extreme scores, 9 low-anxious and 11 high-anxious individuals were selected. Age was well matched across the two groups ( $M=19$ years, $S D=0.6$ for the high-anxious group; $M=20$ years, $S D=2.2$ for the low-anxious group). The gender ratios were $44 \%$ females for the low-anxious group and $64 \%$ females for the high-anxious group (Yates corrected $\left.\chi^{2}=0.17, p=.68\right)$. Mean scores in the Acrophobia Questionnaire were $0.44(S D=0.27)$ for the low-anxious group and $2.66(S D=1.04)$ for the high-anxious group, $t(18)=6.22$, $p<.001$.

\section{Procedure}

After physiological sensors and a head-mounted display were attached, participants stood on a wooden platform (size about $2 \mathrm{ft} \times 1 \mathrm{ft}$, height above floor about $8 \mathrm{in}$.) in a 

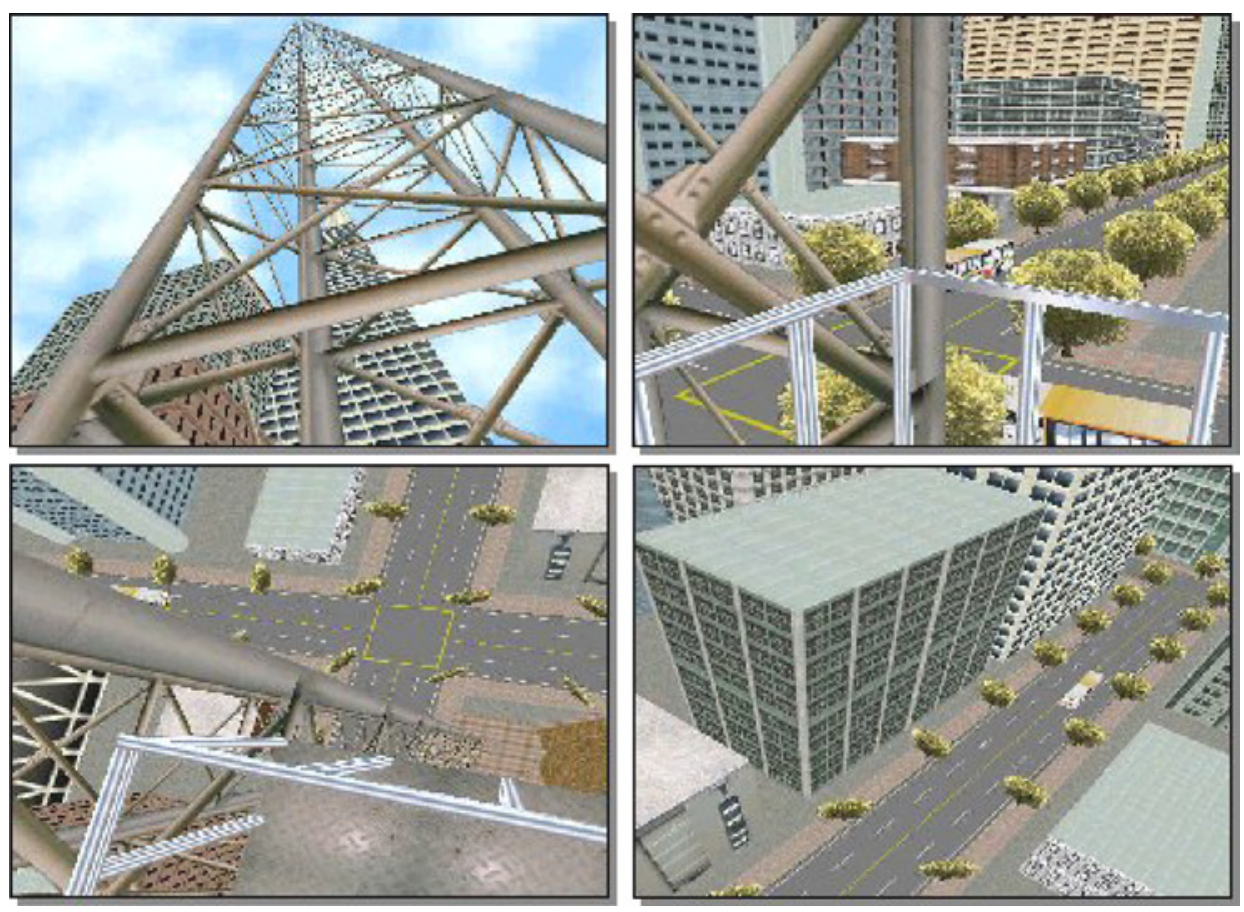

Fig. 1. Snapshots from the VR height exposure world.

darkened room and first viewed a neutral film (baseline, $5 \mathrm{~min}$ ). Thereafter, they experienced a VR simulated height environment through visual and auditory stimuli delivered through the head-mounted display for $14 \mathrm{~min}$. The VR exposure consisted of a scaffolding elevator simulation. In VR, subjects were standing on the platform of an open cabin attached to a metal tower of about $100 \mathrm{~m}$ height situated in a city environment. The experimenter controlled the movement of the open platform and raised it higher in five steps (1: bottom, 5: top), and lowered it in two steps (6: halfway down, 7: bottom) at a constant speed. The subjects were instructed to move their head and look around. The head-mounted display sensed rotation and tilt and adjusted the display accordingly. Subjects could also look down to the wide metal mesh they were standing on and beneath the platform. Figure 1 shows snapshots from the VR height exposure world as seen by the participants through their head-mounted display, depending on the stage of the exposure (not under their control) and the direction of their head (under their control).

\section{Self-Report Measures}

At the end of each step (every 2 min), participants gave oral reports on their fear on an 11-point scale from 0: "no anxiety at all" to 10: "extreme anxiety." After completing the exposure session, participants filled out a self-report questionnaire to assess their presence in the virtual environment. The questionnaire comprised eight items regarding the participants' presence, six of which were taken from Usoh, Catena, Arman, and Slater (2000). The items 
concerned the participants' sense of being in the virtual environment, for example, the extent to which virtual reality was "real" for them, whether they thought of the virtual environment more as images they saw or more as a situation they were in, how much attention they paid to the real world, and the extent to which they focused on the virtual task. In addition, to evaluate potential physiological side effects of the VR exposure, subjects rated how nauseous, strained, and dizzy they felt, and whether they had a headache or not. Each item was rated on a scale from 1 to 7 . Mean item scores on these two scales were evaluated statistically.

\section{Physiological Measures}

Continuous physiological recordings were made using a custom 12-channel SA Instruments (San Diego, CA) bioamplifier. Physiological channels were A/D converted, sampled at $400 \mathrm{~Hz}$, and simultaneously streamed to disk and displayed to PC monitor using custom laboratory software. Later, customized analysis software (Wilhelm, Grossman, \& Roth, 1999) was applied to physiological data reduction, artifact control, and computation of average physiological scores for each participant. Values from ectopic or other kinds of abnormal beats were deleted and replaced by linearly interpolated values. For the primary variables HR and SCL, as well as for the other exploratory variables, mean values were calculated for the seven 120-s intervals during the 14-min exposure session, as well as for the last $2 \mathrm{~min}$ of the 5-min baseline. Measures of physiological activation were chosen to comprehensively assess response systems known to be involved in emotional responding. Many sensors were attached to the left hand. To reduce movement artifact (subjects were standing throughout the session) the left hand of the subjects was attached to the chest and immobilized using a specially designed cloth.

Data from the following channels were used in the analysis reported here: (1) A standard Lead-II electrocardiogram (three electrodes attached to the chest); the signal was reduced using an R-wave detection algorithm, edited for artifacts, converted to 0.25s epochs using weighted averaging, and stored as HR (in bpm). (2) Skin conductance level (two $\mathrm{Ag} / \mathrm{AgCl}$ electrodes placed on the palmar surface of the middle phalanges of digits 3 and 4 of the left hand); a constant $0.5 \mathrm{~V}$ was applied across the electrodes; the signal was analyzed as mean SCL $(\mu \mathrm{S})$ after editing out movement artifacts. (3) The arterial pressure wave form was obtained from the third finger of the non-dominant hand by means of the Finapres ${ }^{\mathrm{TM}} 2300$ (Ohmeda, Madison, WI) system. Beat-to-beat systolic, diastolic and true mean blood pressures (in $\mathrm{mmHg}$ ) were calculated, and pulse pressure (systolic minus diastolic, in $\mathrm{mmHg}$ ) and rate-pressure product $(\mathrm{HR} \times$ systolic blood pressure, in bpm mmHg) computed. (4) Pulse wave forms were measured with a plethysmograph transducer attached to the left ear lobe. Pulse wave transit time to the ear (in ms) was calculated as the time interval between the $R$ wave of the ECG and the steepest upstroke of the peripheral pulse. (5) A thermistor applied to the tip of the small finger on the non-dominant hand measured finger temperature (in ${ }^{\circ} \mathrm{C}$ ) as a measure of peripheral vasoconstriction. (6) Respiratory pattern was measured using two bellows placed around the upper thorax and the abdomen, attached to a respiratory pneumograph device (James Long Company, Caroga Lake, NY). Calibration against $800 \mathrm{ml}$ fixed-volume bags was accomplished by the least-squares method (Chadha et al., 1982). Respiratory rate (in breaths per minute), tidal volume (in $\mathrm{ml}$ ), and their product, minute ventilation (in 
$1 / \mathrm{min}$ ) as a measure of hyperventilation often associated with acute anxiety (Alpers et al., 2005) were calculated breath-by-breath using customized programs. As measures of central respiratory drive and timing mechanisms that are often related to anxiety, inspiratory flow rate (in $\mathrm{ml} / \mathrm{s}$ ) and duty cycle (without units) were calculated (Gautier, 1980). As a measure of respiratory instability that has previously been found to be related to panic diagnosis and acute anxiety (Alpers et al., 2005; Wilhelm, Gerlach, \& Roth, 2001; Wilhelm, Trabert, \& Roth, 2001a, 2001b), tidal volume variability was calculated as the root mean square successive difference (RMSSD) of tidal volumes (in $\mathrm{ml}$ ).

Because HR is dually controlled by the sympathetic and parasympathetic branch of the autonomic nervous system, we assessed certain other measures linked more directly to their activity to explore the mechanisms for HR changes. For measures of HR variability, ECG data were converted to 120 -s segments of instantaneous $R-R$ intervals with a resolution of $4 \mathrm{~Hz}$. To estimate the power spectral densities, the segments were linearly detrended, Hanning windowed, and subjected to a fast Fourier transform using Welch's algorithm (Welch, 1967). Following established criteria, respiratory sinus arrhythmia (RSA) as a measure of parasympathetic cardiac control was computed by integrating over the high frequency (HF) spectral component of $R-R$ intervals at $0.15-0.40 \mathrm{~Hz}$ (in $\mathrm{ms}^{2}$ ). Low frequency (LF) power was computed by integrating the power spectrum over $0.04-0.14 \mathrm{~Hz}$ (in $\mathrm{ms}^{2}$ ). LF power has been linked to blood pressure regulation and is influenced by a variety of sympathetic and parasympathetic afferent and efferent factors (Berger, Saul, \& Cohen, 1989). Both measures were log-transformed for normalization.

\section{Statistical Analysis}

In a first step, we examined the 3 primary dependent variables self-reported anxiety, $\mathrm{HR}$, and SCL in a 2 (Group: low-anxious vs. high-anxious) $\times 8$ (Time: baseline and seven exposure steps) repeated measures analysis of variance (ANOVA). A significant interaction between the factors Time and Group was considered evidence for differential reactivity to the VR exposure between low- and high-anxious participants. All of the variables were normally distributed. Cell sizes were too small to meaningfully analyze effects of gender.

In a second step, we computed effect size parameters relating to responses in the 3 primary and 15 secondary variables for descriptive purposes, since they are a straightforward characterization of the clinical utility of a measure. Due to technical problems, data of the respiration channels could not be included in the analysis for one of the high-anxious subjects. For these analyses, we used a reactivity (or change) score computed for each variable by averaging the mean values for the two highest levels on the elevator (Steps 4 and 5) and subtracting the baseline value. As a quantification of the strength of group discrimination for different measures during VR exposure, an effect size index $d$ for independent samples (Cohen, 1988) was computed as: $d_{1}=$ (mean reactivity score of high-anxious - mean reactivity score of low-anxious)/pooled $S D$. Positive scores indicated greater increases in the high-anxious group than in low-anxious group. In addition, for each group separately, parallel indices $d_{2}$ and $d_{3}$ for dependent samples were computed to assess the strength of response of different measures to the exposure: $d=$ (peak exposure value - baseline value)/SD. $S D$ was computed as the standard deviation of change scores. Positive scores indicated increases, negative scores decreases from baseline to exposure. These statistics take into account within-group error variance and are independent of sample size. In addition, 
discriminant analyses were performed to compute the percentage correct identification of individuals into high-anxious group versus low-anxious group based on change scores of each primary measure.

Correlational analyses were conducted for exploratory reasons using Pearson's product moment correlation. To assess the effect of the participant's presence in the virtual environment on their self-reported and physiological responses to the exposure, all of the reactivity scores were correlated with immersion scores. In addition, correlations between all of the dependent measures and the scores of the Acrophobia Questionnaire were computed. All correlations were computed separately within the low- and the high-anxious group.

To examine differential effects of VR and real-life exposure on self-ratings of anxiety, SCL and HR, data of 11 phobics and 9 controls were randomly selected from a previously collected flight phobia exposure data set (Wilhelm \& Roth, 1998) and compared with the data of the current sample. Change scores of the anxiety self-report and of HR and SCL of the two studies were standardized using $z$-transformation to make the metrics comparable (e.g., different size of electrodes and amplifier technology produce different values of SCL across studies), and subjected to ANOVAs with the factors Study (VR, real-life) and Group (high-anxious vs. low-anxious). Significance level for alpha was set to .05 in all analyses.

\section{RESULTS}

\section{Self-Reported Anxiety and Other Experiential Measures}

All 20 subjects completed all 7 exposure steps. Figure 2 shows mean levels of selfreported anxiety for low- and high-anxious subjects at baseline and during the exposure. For this measure, ANOVA results revealed significant main effects for the factors Group $(F(1,18)=13.55, p<.002)$ and Time $(F(7,126)=22.23, p<.001)$. As indicated by a significant Time $\times$ Group interaction $(F(7,126)=4.84, p<.001)$, self-reported anxiety in response to exposure increased over time more substantially in the high-anxious group than in the low-anxious group, validating that the experimental procedure successfully
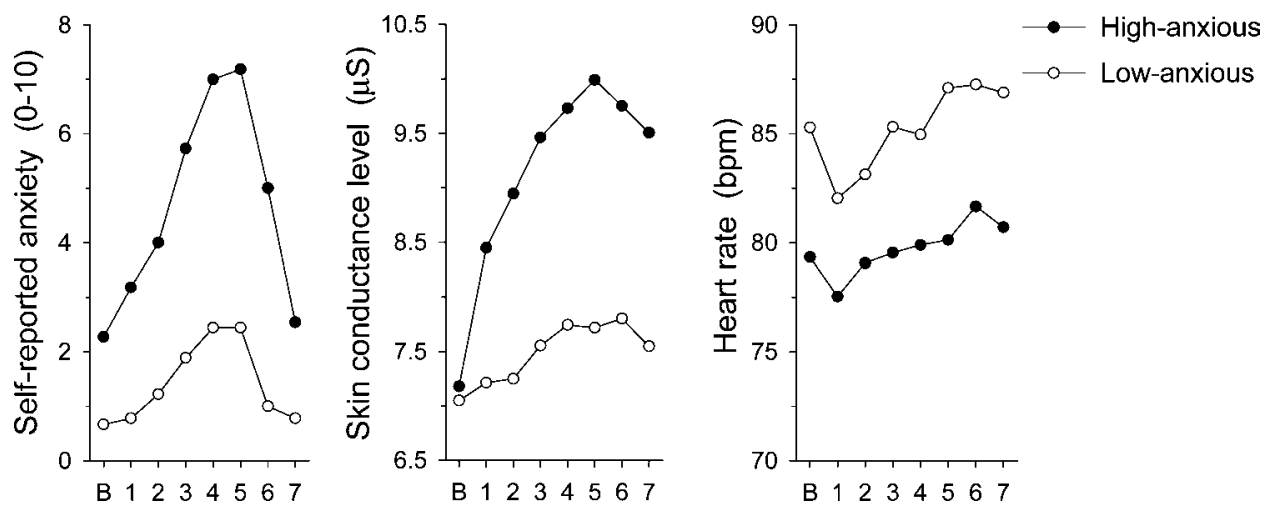

Fig. 2. Responses to the VR exposure in self-reported anxiety, SCL and HR for the high- and low-anxious groups. B: baseline, 1-7: steps 1-7 during the elevator exposure. 
induced high levels of fear in high-anxious participants. Mean immersion scores were 4.26 $(S D=0.73)$ for the high-anxious group and $3.26(S D=0.86)$ for the low-anxious group. This difference was significant, with $t(18)=2.81, p<.02$. Scores on the physiological side effect scale were significantly higher for the high $(M=3.14, S D=2.12)$ than for the low-anxious group $(M=1.36, S D=0.64), t(18)=2.41, p<.03$.

\section{Physiological Measures}

With regard to the primary physiological variables, only reactivity in SCL differed between the two study groups. Following the pattern of self-reported anxiety, a significant main effect for the factor Time $(F(7,126)=8.63, p<.001)$, as well as a significant Group $\times$ Time interaction $(F(7,126)=2.80, p<.01)$ were found, indicating stronger SCL increases in response to exposure in high-anxious participants than in low-anxious participants (Fig. 2). The main effect for Group was not significant. As illustrated in Fig. 2, HR increased slightly during exposure within both study groups, after an initial deceleration at the beginning of the exposure. Accordingly, we found a significant main effect for the factor Time, with $F(7,126)=6.76, p<.001$. Unexpectedly, high-anxious subjects in general showed somewhat lower HRs than low-anxious participants. This effect was not significant $(F(1,18)=1.09, p=.31)$, and neither was the interaction between Group and Time $(F(7,126)=0.65, p=.72)$.

\section{Discriminatory Power of Variables}

Discriminant analyses classified $75 \%$ of cases correctly based on self-reported anxiety reactions to VR exposure, $70 \%$ based on SCL reactions, and only $55 \%$ based on HR reactions (chance level $=50 \%$ ). Table I shows that all of the physiological measures, except the electrodermal measure SCL, had small effect sizes $(d<0.45)$ for group discrimination (row $d_{1}$ ). As is typical for exposure studies, the largest effect size was found for the selfreport measure of anxiety. The data also show that for reactivity within both groups (rows $d_{2}$ and $d_{3}$ ), effect sizes were highest for self-ratings of anxiety, with a particularly high effect size for the high-anxious group. HR did not distinguish exposure from baseline in either of the two groups, whereas a very large effect size for SCL was found for the highanxious group. As is typical in such studies, a number of physiological measures showed about equally strong reactivity for both groups (respiratory rate, systolic blood pressure, rate pressure product), indicating that they were related to anxiety-nonspecific aspects of the procedure. For example, both groups had to immerse themselves in the VR simulation and increase their mental effort from the baseline to the exposure task to comply with the instructions.

\section{Correlational Analyses}

To examine potential neural mechanisms for the absence of HR reactivity in the high-anxious individuals, we computed the correlation between their HR and RSA change scores. We found a highly significant negative correlation, $r=-.84, p<.002$, indicating that the absence of HR reactivity in the anxious group was probably related to increased 
Table I. Effect sizes for reactivity scores (elevator peak minus baseline) for primary and secondary self-report and physiological measures

\begin{tabular}{lrrr}
\hline & \multicolumn{1}{c}{$d_{1}$} & \multicolumn{1}{c}{$d_{2}$} & \multicolumn{1}{c}{$d_{3}$} \\
\hline Primary measures & & & \\
$\quad$ Self-reported anxiety & 1.17 & 1.88 & 0.76 \\
Heart rate & -0.01 & 0.12 & 0.12 \\
Skin conductance level & 0.90 & 1.53 & 0.28 \\
Secondary measures & & & \\
Systolic blood pressure & 0.05 & 0.34 & 0.41 \\
Diastolic blood pressure & -0.17 & 0.19 & 0.45 \\
Mean blood pressure & -0.02 & 0.28 & 0.38 \\
Pulse pressure & 0.33 & 0.37 & 0.22 \\
Rate pressure product & 0.31 & 0.54 & 0.40 \\
Ear pulse transit time & -0.44 & -0.25 & 0.22 \\
Finger temperature & 0.20 & 0.10 & -0.24 \\
Respiratory sinus arrhythmia & 0.41 & 0.31 & -0.09 \\
Low frequency power & 0.32 & 0.44 & 0.42 \\
Respiratory rate & 0.31 & 0.93 & 0.73 \\
Tidal volume & -0.26 & -0.30 & 0.18 \\
Minute ventilation & -0.31 & 0.26 & 0.61 \\
Inspiratory flow rate & -0.37 & 0.17 & 0.69 \\
Respiratory duty cycle & 0.24 & 0.73 & 0.27 \\
Tidal volume variability & -0.21 & -0.23 & 0.20 \\
\hline
\end{tabular}

Note. $d_{1}$ : differential reactivity between high- and low-anxious groups; $d_{2}$ : reactivity within high-anxious group; $d_{3}$ : reactivity within lowanxious group. Absolute values for effect sizes of 0.2 are generally regarded as small, 0.5 as medium, and 0.8 as large. The sign indicates the direction of the effect (see "Methods" section).

activity in the parasympathetic system. Within the high-anxious group, scores on the Acrophobia Questionnaire were strongly associated with electrodermal reactivity during VR height exposure $(r=.68, p<.02)$, but not with HR reactivity $(r=.27, p=.41)$. Selfreported anxiety change scores in this group were correlated with the degree of immersion $(r=.51)$, but probably because of the low sample size, this was not significant $(p=.11)$. No physiological reactivity variable was associated with immersion $(p s>.3)$.

\section{Comparison with In-Vivo Exposure Study}

An ANOVA with the factors Study (VR vs. real-life) and Group (high-anxious vs. lowanxious) with standardized self-reported anxiety reactivity as dependent variable verified that in both studies high-anxious subjects reacted with more anxiety than low-anxious subjects, as reflected by a significant main effect for $\operatorname{Group}(F(1,36)=42.76, p<.001)$. Yet, we also found a marginally significant Group $\times$ Study interaction $(F(1,36)=3.22$, $p=.08$ ), pointing to somewhat higher increases of subjective anxiety in flight phobics during in-vivo exposure than in high-anxious participants in the VR procedures.

Figure 3 shows standardized HR and SCL reactions to exposure in high- and lowanxious subjects of the VR and the in-vivo exposure study. An ANOVA with standardized SCL reactivity as dependent variable and the factors Group (high-anxious vs. lowanxious) and Study (VR vs. real-life) showed no significant Group $\times$ Study interaction effect $(F(1,36)=1.09, p=.30)$, but a significant Group main effect, indicating that independent of the type of exposure, high-anxious participants showed strong electrodermal 

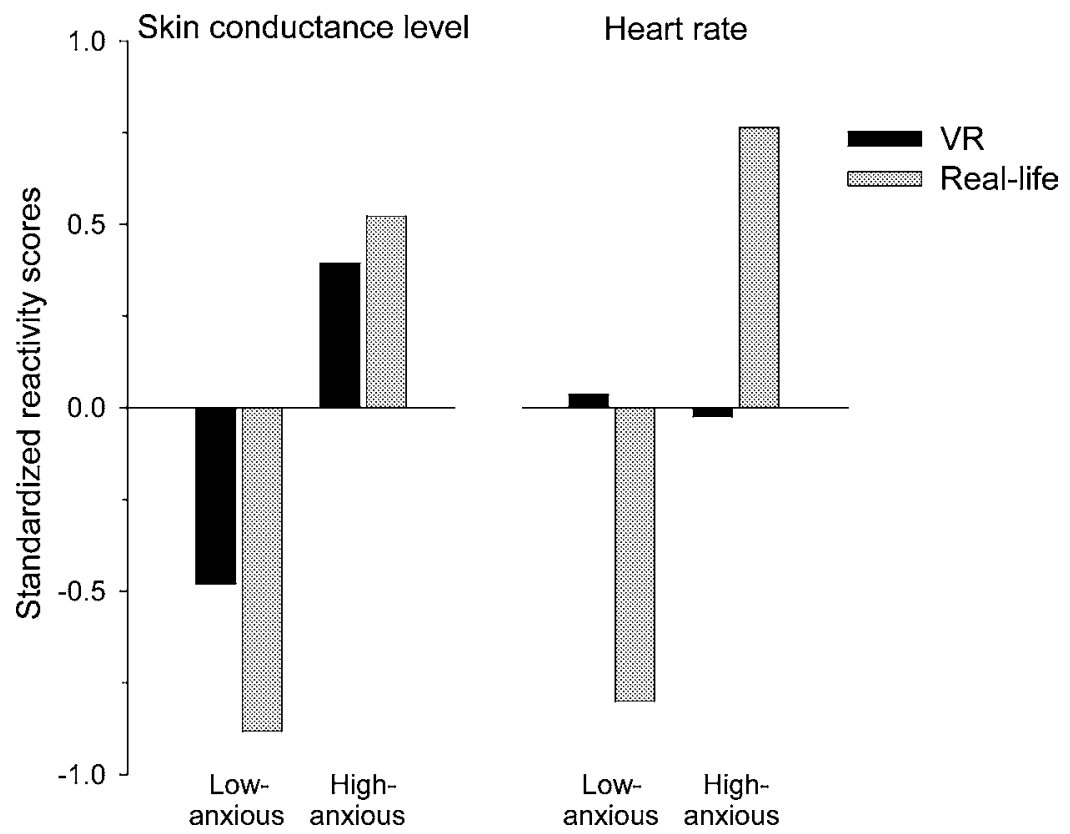

Fig. 3. Standardized SCL and HR reactions (peak exposure minus baseline) to VR exposure in high- and low-anxious subjects comparing the current VR and a previous real-life situational phobia exposure study (see text).

response when confronted with feared situations $(F(1,36)=20.18, p<.001)$. In contrast, a similar analysis for standardized HR reactivity as dependent variable revealed a highly significant interaction effect $(F(1,36)=7.30, p<.02)$, indicating HR increases in high-anxious participants of the real-life but not of the VR exposure study.

\section{DISCUSSION}

Recent advances in VR technology have led to concerted efforts to apply this technology to the treatment of anxiety disorders (Anderson, Jacobs, \& Rothbaum, 2004; Maheu et al., 2005; Riva \& Wiederhold, 2002). These treatments have been shown to be effective for certain situational phobias, including acrophobia and fear of flying (Krijn et al., 2004), but it is not clear whether the mechanisms underlying these VR-treatment based therapeutic effects are similar to those underlying conventional in-vivo exposure.

In the present study, we examined VR simulation of heights with individuals who were high or low in acrophobia. Findings indicated that although anxiety self-reports seemed to suggest that VR exposure led to high levels of anxiety, the only physiological measure that discriminated well between the two study groups was skin conductance, in stark contrast to typical response patterns during in-vivo exposure. The absence of HR responsiveness to VR exposure is particularly worth noting, as HR has repeatedly been the most potent physiological anxiety measure in real-life exposure studies with individuals suffering from specific phobias of the situational type (Alpers et al., 2005; Wilhelm \& Roth, 1998), and 
is believed to be an important indicator of emotional processing during exposure therapy (Foa \& Kozak, 1986; Foa \& Kozak, 1998; Foa \& McNally, 1996).

\section{Selective BIS Activation During VR Exposure}

Our findings are in accordance with Fowles' (1988) motivational theory. Whereas VR exposure apparently mainly activates what Fowles refers to as the behavioral inhibition system (BIS), in-vivo exposure activates both the BIS and the behavioral activation system (BAS). We speculate that the crucial difference between VR and in-vivo contexts may be that the real-life exposure study made use of ambulatory monitoring systems that allow participants to move freely during exposure and, thus, at least theoretically, to show active avoidance, allowing for the BAS to be activated. In contrast, participants of the current study were enclosed in a small darkened testing room, wore a VR headset, and had various sensors attached to an immobilized arm. While standing on a small platform they were connected to stationary amplifiers and thus could not move without restraint. Within the VR world, they were not in control over the movement of the elevator platform. Apparently, one or more of these contextual factors have hindered the triggering of a cardiovascular fight-flight response, which is often conceptualized as an evolutionarily evolved preparation mechanism for physical work.

The lack of HR activation during VR exposure might further be associated with VRspecific effects, such as the influences of visual attention or immersion, i.e., the feeling to be present in a virtual environment. Immersion may be seen as an essential condition for successful VRET, as it is necessary for anxiety to occur. In addition, fearful individuals immerse better into their phobia-matched VR simulations than non-fearful controls (Robillard, Bouchard, Fournier, \& Renaud, 2003). In our study, immersion was elevated in the high-anxious individuals, as indicated by their scores in the questionnaire administered after exposure. Moreover, replicating previous studies (Regenbrecht, Schubert, \& Friedman, 1998; Slater, Pertaub, \& Steed, 1999), for the high-anxious group, we found a trend for a correlation between high levels of immersion and high levels of self-reported anxiety during exposure, indicating that at least subjectively experienced levels of fear may be related to presence in the virtual environment. Yet, neither SCL nor any other physiological measure correlated with immersion. This might have to do with the fact that immersion is closely related to attention processes, i.e., the need to focus one's attention to the scene presented via a head-mounted display.

Focusing one's attention on a virtual environment might result in physiological changes that are specifically related to visual attention, and which differ from physiological responses to a real environment. Berntson and colleagues (Berntson, Cacioppo, \& Fieldstone, 1996) reported significant increases of cardiac parasympathetic activity in participants focusing their attention on slides showing visual illusions. A similar attention effect might have appeared in the present study. That is, cardiac parasympathetic activity during VR exposure might have inhibited increases of HR. This assumption is supported by a strong negative correlation between RSA and HR reactivity scores within the high-anxious group. Similarly, in our study of socially anxious individuals attending to physical touch, RSA increased and HR decreased while subjects reported high levels of anxiety (Wilhelm, Kochar, Roth, \& Gross, 2001). In this study, the touch also induced physical constraint that may have reduced BAS activation. 


\section{VR Versus In-Vivo Exposure}

A direct comparison of our VR data with that of a previous in-vivo exposure study (Wilhelm \& Roth, 1997, 1998) indicated that standardized HR reactivity was equivalent between phobics and controls during VR exposure, but differed during in-vivo exposure. Standardized SCL reactivity patterns, on the other hand, did not differ between studies. Thus, in contrast to in-vivo exposure studies, this VR exposure study identified SCL, but not $\mathrm{HR}$, as a reliable physiological measure that might be used to objectively track anxiety and treatment progress during VR exposure. This is further underlined by the weak classification accuracy of $55 \%$ (which is almost chance level) for HR in the current study, in contrast to $80 \%$ in the in-vivo exposure study. SCL was about equally discriminative for both studies (70\% vs. $65 \%$ ).

Cardiovascular measures in our study besides HR (which is under dual control) are almost exclusively influenced by sympathetic efferents. SCL is also activated by sympathetic efferents but through other pathways and thus not necessarily tied to cardiovascular control (Boucsein, 1992). As indicated by the effect sizes for the discrimination between the two groups, physiological reactivity to exposure attributable to anxiety was low for cardiovascular and respiratory measures, in stark contrast to subjective selfratings of anxiety, which strongly rose during exposure. Discordance between subjective self-reports and physiological measures of anxiety, as well as between different physiological systems is an often observed clinical phenomenon that is not well understood (Grossman, Wilhelm, Kawachi, \& Sparrow, 2001; Hodgson \& Rachman, 1974; Mauss, Levenson, Wilhelm, McCarter, \& Gross, 2005; Mauss, Wilhelm, \& Gross, 2004; Wilhelm, Kochar, et al., 2001). Although researchers and clinicians generally agree with Lang (1968) that the assessment of anxiety should-besides subjective experience measuresinclude physiological and behavioral measures, physiological measures are often neglected. This is not unproblematic because in clinical anxiety physiological disturbances are often a central part of the patient's experiences and one reason to present for treatment (Wilhelm \& Roth, 2001). Our current study highlights the specific value of electrodermal activity as an objective anxiety index during VR exposure studies and treatments.

In light of the overall relatively weak physiological changes in response to VR exposure, one might argue that the mechanisms underlying VRET differ from the ones underlying in-vivo exposure. Following the theory by Foa and Kozak (1986), successful exposure therapy should elicit enough fear for habituation processes to occur. Our study indicates that during VRET the behavioral inhibition, but not the behavioral activation system is triggered, which might suffice for habituation of relevant fear nodes in the network. On the other hand, it is entirely possible that in VRET, at least initially, cognitive restructuring mechanisms are more important than physiological activation and habituation. Patients going through VRET probably become convinced that they can master fear-inducing situations, and, as a consequence of their heightened self-efficacy expectations, expose themselves to them more in real-life, where physiological activation, habituation, and full restructuring of the fear network can occur. This possibility however needs to be further investigated. In future studies, fMRI or EEG might be used to identify central correlates of VR-induced fear, and help elucidate the CNS mechanisms, including the BIS and BAS, associated with VRET. 


\section{Limitations and Future Directions}

The present study has certain limitations. First, we did not test a clinical sample, our sample size was small, and gender ratios for the high- and low-anxious groups were not fully balanced. Therefore, generalizations of the results, especially pertaining to correlational analyses, should be made with caution. However, scores of the high-anxious subjects of our study in the Acrophobia Questionnaire were much elevated, and self-reports of anxiety point to high levels of anxiety in these subjects during VR exposure. Furthermore, even with the small sample size, our results (e.g., strong increases of SCL but no HR response to exposure) are unambiguous. The comparison of our data with the ones of the flight phobia study by Wilhelm and Roth (1998) could further have been influenced by differences between the experimental settings and type of phobia, as well as by the fact that only one of the two studies tested a clinical sample. Future studies should make direct comparisons between real-life and VR exposure using the same subjects and procedures, rather than comparing studies differing in experimental setting, data reduction procedures, and study populations.

\section{ACKNOWLEDGMENTS}

This research was supported by Grant 105311-105850 from the Swiss National Science Foundation (FHW) and by Grant MH58147 from the National Institutes of Mental Health (JJG). The authors would like to thank Thomas Nguyen for his help with the data collection and Dr. Hartmut Schächinger for helpful suggestions with the statistical analysis.

\section{REFERENCES}

Alpers, G. W., Wilhelm, F. H., \& Roth, W. T. (2005). Psychophysiological assessment during exposure in driving phobic patients. Journal of Abnormal Psychology, 114(1), 126-139.

Anderson, P., Jacobs, C., \& Rothbaum, B. O. (2004). Computer-supported cognitive behavioral treatment of anxiety disorders. Journal of Clinical Psychology, 60(3), 253-267.

Barlow, D. H. (Ed.). (2002). Anxiety and its disorders: The nature and treatment of anxiety and panic (2nd ed.). New York, NY, USA: The Guilford Press.

Berger, R. D., Saul, J. P., \& Cohen, R. J. (1989). Transfer function analysis of autonomic regulation. I. Canine atrial rate response. American Journal of Physiology, 256(1 Pt 2), H142-152.

Berntson, G. G., Cacioppo, J. T., \& Fieldstone, A. (1996). Illusions, arithmetic, and the bidirectional modulation of vagal control of the heart. Biological Psychology, 44(1), 1-17.

Boucsein, W. (1992). Electrodermal activity. New York: Plenum Press.

Chadha, T., Watson, H., Birch, S., Jenouri, G., Schneider, A., Cohn, M., et al. (1982). Validation of respiratory inductive plethysmography using different calibration procedures. American Review of Respiratory Disease, 125(6), 644-649.

Cohen, D. C. (1977). Comparison of self-report and behavioral procedures for assessing acrophobia. Behavior Therapy, 18, 17-23.

Cohen, J. (1988). Statistical power analysis for the behavioral sciences (2nd ed.). Hillsdale: Lawrence Erlbaum Associates.

Foa, E. B., \& Kozak, M. J. (1986). Emotional processing of fear: Exposure to corrective information. Psychological Bulletin, 99(1), 20-35.

Foa, E. B., \& Kozak, M. J. (1998). Clinical applications of bioinformational theory: Understanding anxiety and its treatment. Behavior Therapy, 29(4), 675-690.

Foa, E. B., \& McNally, R. J. (1996). Mechanisms of change in exposure therapy. In R. M. Rapee (Ed.), Current controversies in the anxiety disorders (pp. 229-343). New York: Guilford Press.

Fowles, D. C. (1980). The three arousal model: Implications of Gray's two-factor learning theory for heart rate, electrodermal activity, and psychopathy. Psychophysiology, 17(2), 87-104. 
Fowles, D. C. (1988). Psychophysiology and psychopathology: A motivational approach. Psychophysiology, 25(4), 373-391.

Gautier, H. (1980). Control of pattern of breathing. Clinical Science, 58, 343-348.

Gray, J. A. (1975). Elements of a two-process theory of learning. New York: Academic Press.

Grossman, P., Wilhelm, F. H., Kawachi, I., \& Sparrow, D. (2001). Gender differences in psychophysiological responses to speech stress among older social phobics: Congruence and incongruence between self-evaluative and cardiovascular reactions. Psychosomatic Medicine, 63(5), 765-777.

Hodgson, R., \& Rachman, S. (1974). Desynchrony in measures of fear. Behaviour Research and Therapy, 12, 319-326.

Krijn, M., Emmelkamp, P. M., Olafsson, R. P., \& Biemond, R. (2004). Virtual reality exposure therapy of anxiety disorders: a review. Clinical Psychological Review, 24(3), 259-281.

Lang, P. J. (1968). Fear reduction and fear behavior: Problems in treating a construct. In J. M. Shlien (Ed.), Research in psychotherapy (Vol. 3, pp. 90-103). Washington, DC: American Psychological Association.

Maheu, M. M., Pulier, M. L., Wilhelm, F. H., McMenamin, J. P., \& Brown-Connolly, N. E. (2005). The mental health professional and the new technologies. Mahwah, NJ: Lawrence Erlbaum Associates.

Mauss, I. B., Levenson, R. W., Wilhelm, F. H., McCarter, L., \& Gross, J. J. (2005). The tie that binds? Coherence among emotion experience, behavior, and physiology. Emotion, 5(2), 175-190.

Mauss, I. B., Wilhelm, F. H., \& Gross, J., J. (2004). Is there less to social anxiety than meets the eye? Emotion experience, expression, and bodily responding. Cognition and Emotion, 18(5), 631-662.

Regenbrecht, H. T., Schubert, T. W., \& Friedman, F. (1998). Measuring the sense of presence and its relation to fear of heights in virtual environments. International Journal of Human-Computer Interaction, 10, 233-249.

Riva, G., \& Wiederhold, B. K. (2002). Introduction to the special issue on virtual reality environments in behavioral sciences. IEEE Transactions on Information Technology in Biomedicine, 6(3), 193-197.

Robillard, G., Bouchard, S., Fournier, T., \& Renaud, P. (2003). Anxiety and presence during VR immersion: A comparative study of the reactions of phobic and non-phobic participants in therapeutic virtual environments derived from computer games. Cyberpsychology and Behavior, 6(5), 467-476.

Rothbaum, B. O., Hodges, L., Smith, S., Lee, J. H., \& Price, L. (2000). A controlled study of virtual reality exposure therapy for the fear of flying. Journal of Consulting and Clinical Psychology, 68(6), 1020-1026.

Rothbaum, B. O., Hodges, L. F., Kooper, R., Opdyke, D., Williford, J. S., \& North, M. (1995). Effectiveness of computer-generated (virtual reality) graded exposure in the treatment of acrophobia. American Journal of Psychiatry, 152(4), 626-628.

Slater, M., Pertaub, D., \& Steed, A. (1999). Public speaking in virtual reality: Facing an audience of avatars. IEEE Computer Graphics and Applications, 19, 6-9.

Usoh, M., Catena, E., Arman, S., \& Slater, M. (2000). Using presence questionnaires in reality. Presence: Teleoperators and Virtual Environments, 9(5), 487-503.

Welch, P. D. (1967). The use of fast Fourier transform for the estimation of power spectra: A method based on time averaging over short modified periodograms. IEEE Transactions on Audio and Electroacoustics, 15, $70-73$.

Wiederhold, B. K., Jang, D. P., Kim, S. I., \& Wiederhold, M. D. (2002). Physiological monitoring as an objective tool in virtual reality therapy. Cyberpsychology and Behavior, 5(1), 77-82.

Wilhelm, F. H., Gerlach, A. L., \& Roth, W. T. (2001). Slow recovery from voluntary hyperventilation in panic disorder. Psychosomatic Medicine, 63, 638-649.

Wilhelm, F. H., Grossman, P., \& Roth, W. T. (1999). Analysis of cardiovascular regulation. Biomedical Sciences Instrumentation, 35, 135-140.

Wilhelm, F. H., Kochar, A. S., Roth, W. T., \& Gross, J. J. (2001). Social anxiety and response to touch: incongruence between self-evaluative and physiological reactions. Biological Psychology, 58, 181-202.

Wilhelm, F. H., \& Roth, W. T. (1997). Acute and delayed effects of alprazolam on flight phobics during exposure. Behaviour Research and Therapy, 35(9), 831-841.

Wilhelm, F. H., \& Roth, W. T. (1998). Taking the laboratory to the skies: Ambulatory assessment of self- report, autonomic, and respiratory responses in flying phobia. Psychophysiology, 35(5), 596-606.

Wilhelm, F. H., \& Roth, W. T. (2001). The somatic symptom paradox in DSM-IV anxiety disorders: Suggestions for a clinical focus in psychophysiology. Biological Psychology, 57(1-3), 105-140.

Wilhelm, F. H., Trabert, W., \& Roth, W. T. (2001a). Characteristics of sighing in panic disorder. Biological Psychiatry, 49(7), 606-614.

Wilhelm, F. H., Trabert, W., \& Roth, W. T. (2001b). Physiological instability in panic disorder and generalized anxiety disorder. Biological Psychiatry, 49(7), 596-605. 